PROCEEDINGS OF THE

AMERICAN MATHEMATICAL SOCIETY

Volume 126, Number 3, March 1998, Pages 863-865

S $0002-9939(98) 04384-6$

\title{
A NOTE ON THE DENSITY OF $s$-DIMENSIONAL SETS
}

\author{
JAMES FORAN
}

(Communicated by James West)

\begin{abstract}
Sets in Euclidean spaces which are measurable with respect to Hausdorff $s$-dimensional measure with $0<s<1$ are shown to have an at most countable set of points where the exact $s$-density exists and is finite and non-zero.
\end{abstract}

The purpose of this note is to give a slight improvement to the result of Marstrand [2] which states that for $0<s<1$, the set of points of an $s$-set at which the exact density exists and is finite is of $s$-measure 0 . This result was given a simpler proof by Falconer [1, p. 55-56] and the theorem below uses his notation and definitions. Indeed, this stronger result is patterned on his proof.

Definitions. $B_{r}(y)=\{x: d(x, y) \leq r\}$ is the closed ball about $x$ of radius $r$.

$H_{\delta}^{s}(E)=\inf \sum_{i=1}^{\infty}\left|U_{i}\right|$ where the inf is over all countable covers of $E$ with $\operatorname{diam}\left(U_{i}\right) \leq \delta$.

$H^{s}(E)=\lim _{\delta \rightarrow 0} H_{\delta}^{s}(E)$ is the Hausdorff outer measure of $E$.

$D^{s}(E, x)=\lim _{r \rightarrow 0} H^{s}\left(E \cap B_{r}(x)\right) /(2 r)^{s}$ is the density of $E$ at $x$ provided the limit exists.

The upper density and lower density are defined using the limsup and liminf respectively.

Theorem. Suppose $E$ is an s-measurable set in Euclidean $n$-dimensional space with $0<s<1$. Then the set of points $x$ where the density $D^{s}(E, x)$ exists and is finite and non-zero is an at most countable set of points.

Proof. Given a natural number $k$ and $0<s<1$, suppose there is a set $E$ in a Euclidean space for which the set of points $A$ where $D^{s}(E, x)$ is finite and non-zero is an uncountable set. Let $A_{j}$ be the set of all $x \in A$ for which $r<1 / j$ implies $(2 r)^{s} / j<H^{s}\left(E \cap B_{r}(x)\right)<(2 r)^{s} \cdot j$. Since $A=\bigcup A_{j}$ there is a natural number $N$ so that $A_{N}$ is uncountable and thus there is a point $y \in A_{N}$ which is an accumulation point of $A_{N}$. Let $0<\eta<1$ and let $x \in A_{N}$ with $d(x, y)=r$ and $r(1+\eta)<1 / N$. Let $A_{r, \eta}(y)=B_{r(1+\eta)}(y) \backslash B_{r(1-\eta)}(y)$, the annulus centered at $y$. Note (as in [1]) that

$$
\begin{aligned}
(2 r)^{-s} H^{s}\left(E \cap A_{r, \eta}(y)\right) & =(2 r)^{-s} H^{s}\left(E \cap B_{r(1+\eta)}(y)\right)-(2 r)^{-s} H\left(E \cap B_{r(1-\eta)}(y)\right) \\
& \rightarrow D^{s}(E, y)\left((1+\eta)^{s}-(1-\eta)^{s}\right) .
\end{aligned}
$$

Received by the editors September 14, 1996.

1991 Mathematics Subject Classification. Primary 28A78.

(C)1998 American Mathematical Society 
Then, since $B_{r \eta / 2}(x) \subset A_{r, \eta}(y)$, it follows that

$$
r^{s} \eta^{s} / N<H^{s}\left(E \cap B_{r \eta / 2}(x)\right) \leq H^{s}\left(E \cap A_{r, \eta}(y)\right)
$$

and on dividing the terms of this inequality by $(2 r)^{s}$ and letting $r$ approach 0 , it follows that

$$
\eta^{s} /\left(N \cdot 2^{s}\right) \leq D^{s}(E, y)\left((1+\eta)^{s}-(1-\eta)^{s}\right)=D^{s}(E, y)\left(2 s \eta+O\left(\eta^{2}\right)\right)
$$

which is impossible for sufficiently small $\eta$. This contradiction shows that each $A_{j}$ consists of isolated points and from this it also follows that $A$ is an at most countable set.

To complete this picture, suppose that $\left\{x_{i}\right\}$ is a sequence of points in a Euclidean space and $\left\{d_{i}\right\}$ is a sequence of extended real numbers with $0<d_{i} \leq \infty$. The following example shows that it is possible for a set to have exact $s$-density $d_{i}$ at each point $x_{i}$.

Example. Given points $x_{i}$ in Euclidean $k$-dimensional space, numbers $d_{i}$ with $0<d_{i} \leq \infty$ and $s \in(0,1)$, there is a set $E$ of finite $s$-measure so that $D^{s}\left(E, x_{i}\right)=d_{i}$.

Construction. The set will be constructed on a sequence of line segments parallel to one of the axes. First, let $0<d<\infty$ and $s \in(0,1)$ be given. Place a closed set $X_{N}$ of $s$-measure $2^{s} \cdot d\left(N^{-s}-(N+1)^{-s}\right)$ in each interval $\left((N+1)^{-1}, N^{-1}\right)$. Then $E_{d}=\bigcup X_{i} \cup\{0\}$ is a closed set. Since $2^{s} \cdot d \cdot \sum_{n=N}^{\infty}\left(1 / n^{s}-1 /(n+1)^{s}\right) /(2 r)^{s}=$ $d \cdot N^{-s} / r^{s}$, if $1 /(N+1) \leq r<1 / N$, it follows that

$$
d \cdot(N+1)^{-s} / r^{s} \leq H^{s}\left(E_{d} \cap(-r, r)\right) /(2 r)^{s} \leq d \cdot N^{-s} / r^{s} .
$$

Since the opposite sides of this inequality approach $d$ as $r$ approaches 0 , the density of $E_{d}$ is $d$ at 0 . If it is desired to have a set $E_{\infty}$ of finite measure and $s$-density $\infty$ at the origin, let $d_{n} \uparrow \infty$ and construct closed sets $X_{N} \subset\left((N+1)^{-1}, N^{-1}\right)$ of $s$-measure $2^{s} \cdot d_{N} \cdot\left(N^{-s}-(N+1)^{-s}\right)$. Then the same calculations show that the set $E_{\infty}=\bigcup X_{i} \cup\{0\}$ has density $\infty$ at 0 . If the $d_{n}$ are chosen so that

$$
\sum d_{n}\left(n^{-s}-(n+1)^{-s}\right)<\infty
$$

then the $s$-measure of the set $E_{\infty}$ will be finite. Note that $E_{d}$ can be considered to be a subset of $E^{k}$ of points on the first coordinate axis with $s$-density $d$ at the origin. Suppose $\left\{x_{i}\right\}$ and $\left\{d_{i}\right\}$ are given. Let $E_{1}=F_{1}=E_{d_{1}}+x_{1}$. Let $r_{1}=1$ and let $r_{n}=\min \left\{2^{-n}, d\left(x_{i}, x_{j}\right): i<j \leq n\right\}$. Suppose $E_{n-1}$ and $F_{n-1}$ have been constructed. Let $\varepsilon_{n}>0$ with $\varepsilon_{n}<r_{n} / 2^{n}$ so that $H^{s}\left(B_{\varepsilon_{n}}\left(x_{n}\right) \cap E_{n-1}\right)<2^{-n} \cdot r_{n+1}^{s}$ and $H^{s}\left(B_{\varepsilon_{n}}(0) \cap E_{d_{n}}\right)<2^{-n} \cdot r_{n+1}^{s}$. Let $F_{n}=\left(E_{d_{n}} \cap B_{\varepsilon_{n}}(0)\right)+x_{n}$ and let $E_{n}=\left(E_{n-1} \backslash B_{\varepsilon_{n}}\left(x_{n}\right)\right) \cup F_{n}$. Then $E=\lim \sup E_{n}$ is the required set. It clearly has finite $s$-measure and if $r>0$ is given with $r_{n+1}\left(1-2^{-n}\right) \leq r<r_{n}\left(1-2^{-n}\right)$, then $H^{s}\left(F_{i} \cap B_{r}\left(x_{i}\right)\right)-2^{-n+1} \cdot r_{n+1}^{s} \leq H^{s}\left(E \cap B_{r}\left(x_{i}\right)\right) \leq H_{s}\left(F_{i} \cap B_{r}\left(x_{i}\right)\right)+2^{-n+1} \cdot r_{n+1}^{s}$. Dividing the terms in this inequality by $(2 r)^{s}$ and letting $r \rightarrow 0$, the two sides of the resulting inequality approach $d_{i}$. It follows that the $s$-density of $E$ at $x_{i}$ is $d_{i}$.

Some natural questions which arise are:

1. Is it possible that the set in the example above be constructed so that it has the exact preassigned $s$-density only at the preassigned points?

2. What kind of structure does the set of points where the $s$-density is infinite have?

3. Is there a generalization for $s>1$; for example, is it possible that the set of points is always of $\sigma$-finite $n-1$ measure where $n-1<s<n$ ? 


\section{REFERENCES}

1. K. J. Falconer, The Geometry of Fractal Sets, Cambridge Univ. Press, 1985. MR 88d:28001

2. J. M. Marstrand, Some fundamental properties of plane sets of fractional dimension, Proc. Lond. Math. Soc. (3) 4 (1954), 257-302. MR 16:121g

Department of Mathematics, University of Missouri-Kansas City, Kansas City, MisSOURI 64110

E-mail address: jforan@cctr.umkc.edu 